\title{
Long-Term Effect of Rigid Foot Orthosis in Children Older Than Six Years With Flexible Flat Foot
}

\author{
Kyo-Jun Youn, $\mathrm{MD}^{1}$, So Young Ahn, $\mathrm{MD}, \mathrm{PhD}^{1}$, Bong-Ok Kim, $\mathrm{MD}, \mathrm{PhD}^{2}$, \\ In Sik Park, BS ${ }^{3}$, Soo-Kyung Bok, MD, PhD ${ }^{1}$
}

\begin{abstract}
${ }^{1}$ Department of Rehabilitation Medicine, Chungnam National University Hospital, Daejeon; ${ }^{2}$ Korea Worker's Compensation \& Welfare Service Daegu Hospital, Daegu; ${ }^{3}$ Korean Pedorthic Institute, Goyang, Korea
\end{abstract}

Objective To evaluate the long-term effect of a custom-made rigid foot orthosis (RFO) in children older than 6 years with pes planus (flat foot).

Methods Medical records of 42 children diagnosed with flexible pes planus who were fitted with RFOs based on the inverted technique and underwent more than four consecutive radiological studies were reviewed. Resting calcaneal stance position (RCSP), anteroposterior talocalcaneal angle, lateral talocalcaneal angle, lateral talometatarsal angle, and calcaneal pitch were initially measured in both feet to evaluate alignment. Followup clinical and radiological evaluations were then performed at 12-18, 24-30, 36-42, and $\geq 48$ months after RFO application. Repeated measures analysis of variance was used to evaluate significant differences.

Results Significant improvements in all radiological indicators and significant progression of RCSP toward the corrective direction were observed after RFO application relative to baseline measurements.

Conclusion According to our findings, RFO can induce significant improvements in calcaneus-related radiographic indices and subsequently improve talus-related radiologic indices.

Keywords Flatfoot, Foot orthoses, Radiology, Child

\section{INTRODUCTION}

Pes planus, or flat foot, is used to describe a foot with a low or absent longitudinal arch. Pediatric pes planus is mild and asymptomatic in most cases. However, in more severe forms, it may present with a variety of symptoms that can lead to severe sequelae and dysfunction [1] such as pelvic malalignment syndrome and subsequent scoliosis [2]. Some children with a flexible flat foot may develop fixed secondary deformities and arthritic changes in the

Received August 7, 2018; Accepted October 17, 2018

Corresponding author: Soo-Kyung Bok

Department of Rehabilitation Medicine, Chungnam National University Hospital, 282 Munhwa-ro, Jung-gu, Daejeon 35015, Korea. Tel: +82-42-3382460, Fax: +82-42-338-2461, E-mail: skbok@cnuh.co.kr

ORCID: Kyo-Jun Youn (http://orcid.org/0000-0002-9632-1890); So Young Ahn (http://orcid.org/0000-0002-7447-0617); Bong-Ok Kim (http://orcid. org/0000-0002-4831-5023); In Sik Park (http://orcid.org/0000-0001-7307-1078); Soo-Kyung Bok (http://orcid.org/0000-0002-8957-2827).

(c) This is an open-access article distributed under the terms of the Creative Commons Attribution Non-Commercial License (http://creativecommons.org/ licenses/by-nc/4.0) which permits unrestricted noncommercial use, distribution, and reproduction in any medium, provided the original work is properly cited. Copyright (๑) 2019 by Korean Academy of Rehabilitation Medicine 
feet as well as various radiologic abnormalities $[3,4]$.

Physiologic changes associated with pes planus can shorten the Achilles tendon and cause morbidity of the posterior tibial ligament. Furthermore, flattening of the foot can directly cause pain, movement disorders, and poor quality of life [5]. Therefore, treatment of flat feet aims to alleviate pain and disability. It generally involves conservative non-surgical options such as orthotic devices, although surgical approaches may be attempted in rare refractory cases [6]. Accordingly, many authors have debated on the use of orthosis for the treatment of flat foot.

Regarding orthosis, Kuhn et al. [3] have reported a significant difference between radiometric measurements obtained before and after the use of a flexible foot orthosis. Kulcu et al. [7] have reported that flat foot does not affect patient's gait pattern. However, these studies included a heterogeneous group of subjects with respect to age. Whitford and Esterman [8] have reported effects of foot orthosis on children with pes planus aged 7-11 years. However, their study period was as short as 12 months. In addition, the authors did not perform radiographic examinations [8]. Bok et al. [9] have reported effects of foot orthosis on radiologic parameters in children aged $\geq 6$ years. However, their period of investigation was as short as 2 years [9]. Pfeiffer et al. [10] have reported an average prevalence rate of $46.3 \%$ in a study of 2-6 years old children. Lin et al. [11] have found that the prevalence of flat foot decreases from $57 \%$ to $21 \%$ with aging in a similarly aged population. Volpon [12] have shown that most infants have a normal vertical height of the medial longitudinal arch between 2 and 6 years and achieve its complete form at the latter age.

For symptomatic patients, treatment with a foot ortho- sis appears to provide benefits in terms of reducing pain and functional deterioration. However, the use of foot orthosis remains controversial in asymptomatic cases as the provision of proper foot support is assumed to inhibit progression to severe structural abnormalities and/or improve radiologic indicators. The purpose of this study was to evaluate long-term effects of rigid foot orthosis (RFO) in children 6 years of age or older. This age group was determined based on findings of the above-mentioned longitudinal studies of children.

\section{MATERIALS AND METHODS}

In this study, medical records of 42 children aged 6 years or older who were diagnosed with flexible flatfoot, fitted with RFOs based on the inverted technique, and participated in more than four consecutive radiological studies were reviewed. Flexible flat foot was defined as the disappearance of the medial posterior arch only upon weight-loading, a resting calcaneal stance position (RCSP) angle of less than $-4^{\circ}$, and satisfaction of one of the following four radiographic measurement criteria: (1) an anteroposterior talocalcaneal angle (APTCA) $>30^{\circ}$; (2) lateral talocalcaneal angle (LTTCA) $>45^{\circ}$; (3) lateral talometatarsal angle (LTTMA) $>4^{\circ}$; or (4) calcaneal pitch (CP) $<20^{\circ}[4,9,13]$. Patients who met the following criteria were excluded: (1) evidence of a fixed-foot deformity; (2) history of intervention (e.g., previous orthosis or surgery); (3) congenital or developmental foot disease; (4) evidence of neuromuscular or central nervous system disease; and/ or (5) injuries requiring a non-weight bearing period at the time of the study. This study received approval from the Institutional Review Board of Chungnam National University Hospital (No. 2015-03-040).
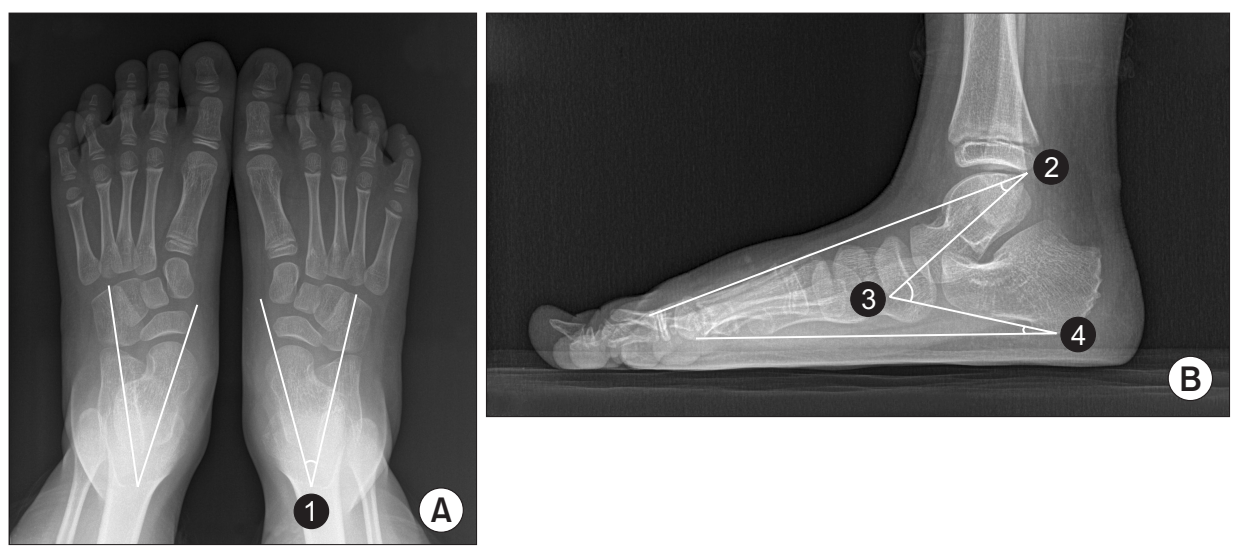

Fig. 1. Representative radiographic images of both feet: (A) anteroposterior view and (B) lateral view indicating (1) anteroposterior talocalcaneal angle, (2) lateral talometatarsal angle, (3) lateral talocalcaneal angle, and (4) calcaneal pitch. 


\section{Resting calcaneal stance position}

The subject was asked to place his foot on the edge of the bed and lay in the prone position on a bed parallel to the ground. The investigator then manually inspected the foot and drew a bisector by placing three dots on the top, middle, and bottom of the heel regardless of fat surrounding the calcaneus. The RCSP was measured when the subject stood on his or her feet placed at a fist-width distance. Angles were determined between the line of heel and the surface.

\section{Radiographic measurement}

All radiological examinations were performed before and after treatment. Participants' feet were examined in both anteroposterior and lateral views while in a standing position. APTCA was defined as the acute angle between the dichotomous line of the calcaneus and talus with the long axis on an anteroposterior radiograph to evaluate rearfoot alignment. LTTCA, LTTMA, and CP were measured in the lateral view to evaluate the medial longitudinal arch of the foot. LTTCA was defined as the angle between the longitudinal axis of the talus and the calcaneus measured on a weight-bearing foot radiograph. LTTMA was defined to the angle between the longitudinal axis of the talus and the first metatarsal bone. $\mathrm{CP}$ was defined as the angle formed by the standing surface and a line from the lower margin of the calcaneus (Fig. 1).

All radiological measurements were independently measured by two rehabilitation physicians to minimize errors. Mean values of all measurements were used for further analyses. Clinical and radiological examinations were performed five times in total: before the use of the RFO and at 12-24, 24-36, 36-48, and 48-60 months after application of the RFO.

\section{Rigid foot orthosis}

RFO is a functional foot orthosis based on Blake's inverted technique. It was developed to control excessive foot pronation $[14,15]$. Specifically, calibration was performed when the calcaneus was everted more than $5^{\circ}$ (i.e., a RCSP of less than $-5^{\circ}$ ). After determining the target RCSP angle, this value was multiplied by five-fold to define the pouring angle. This technique aims to calibrate the heel vertically based on the RCSP, using the medial portion of the calcaneus as the target for correction. Patients were instructed to wear the RFO both indoors and outdoors for at least 8 hours per day.

\section{Statistical analysis}

Mean values and standard deviations of the standing RCSP angle and the four radiographic measurements were calculated and compared between before and after application of the RFO. These values were compared and analyzed using a repeated measures analysis of variance to evaluate degrees of change in measured values over time. Statistical significance was defined at $p$-value $<0.05$.

\section{RESULTS}

This study included 24 boys and 18 girls with a mean age of 9.79 years (range, $6-15$ years). These children chiefly complained of gait abnormalities (8 children, $19.0 \%$ ), foot pain during walking (15 children, 35.7\%), and flat foot itself (19 children, $45.2 \%$ ). Average intervals from baseline until the 2nd, 3rd, 4th, and 5th visits were 18, 26, 40, and 56 months, respectively (Table 1). Serial change and significant results of the RCSP angle and four

Table 1. Basic demographic characteristics of participants $(n=42)$

\begin{tabular}{lc}
\hline \multicolumn{1}{c}{ Characteristic } & Value \\
\hline Age $(\mathrm{yr})$ & $9.79 \pm 2.67$ \\
Sex & \\
$\quad$ Male & 24 \\
$\quad$ Female & 18 \\
\hline Average intervals from baseline (mo) & \\
$\quad$ 1st visit & $18.1 \pm 2.2$ \\
\hline 2nd visit & $26.4 \pm 3.1$ \\
\hline 3rd visit & $40.2 \pm 5.4$ \\
\hline 4th visit & $55.9 \pm 5.7$ \\
Baseline values of radiologic indicators & \\
and RCSP $\left(^{\circ}\right)$ & \\
\hline APTCA & $36.7 \pm 8.1$ \\
\hline LTTCA & $47.0 \pm 6.4$ \\
\hline LTTMA & $16.3 \pm 9.0$ \\
\hline CP & $11.8 \pm 4.2$ \\
\hline RCSP & $-8.3 \pm 5.6$ \\
\hline
\end{tabular}

Values are presented as mean \pm standard deviation.

RCSP, resting calcaneal stance position; APTCA, anteroposterior talocalcaneal angle; LTTCA, lateral talocalcaneal angle; LTTMA, lateral talometatarsal angle; CP, calcaneal pitch. 
radiographic measurements are shown in Fig. 1.

\section{Anteroposterior talocalcaneal angle}

Average APTCA values at baseline, 2nd, 3rd, 4th, and 5 th visits were $36.7^{\circ} \pm 8.1^{\circ}, 36.1^{\circ} \pm 8.5^{\circ}, 29.5^{\circ} \pm 6.9^{\circ}$, $29.1^{\circ} \pm 5.5^{\circ}$, and $28.8^{\circ} \pm 5.1^{\circ}$, respectively. Notably, the 3rd, 4 th, and 5th measurements represented significant improvements over the baseline. However, the most significant difference appeared between the 2nd and 3rd visits.

\section{Lateral talocalcaneal angle}

Average LTTCA measurements at baseline, 2nd, 3rd, 4 th, and 5 th visits were $47.0^{\circ} \pm 6.4^{\circ}, 48.4^{\circ} \pm 7.5^{\circ}, 47.2^{\circ} \pm 4.6^{\circ}$, $45.1^{\circ} \pm 4.3^{\circ}$, and $43.8^{\circ} \pm 4.7^{\circ}$, respectively. Both the 4 th and 5 th measurements represented statistically significant improvements compared to the 2nd measurement and the 5th measurement represented a significant improvement over the baseline.

\section{Lateral talometatarsal angle}

LTTMA measurements at baseline, 2nd, 3rd, 4th, and 5th visits were $16.3^{\circ} \pm 9.0^{\circ}, 15.4^{\circ} \pm 8.4^{\circ}, 8.6^{\circ} \pm 5.2^{\circ}, 8.9^{\circ} \pm 4.5^{\circ}$, and $8.4^{\circ} \pm 4.4^{\circ}$, respectively. The 3rd, 4th, and 5th measurements represented significant improvements over the baseline. However, the most significant difference appeared between the 2nd and 3rd visits.

\section{Calcaneal pitch}

CP measurements at baseline, 2nd, 3rd, 4th, and 5th visits were $11.8^{\circ} \pm 4.2^{\circ}, 14.2^{\circ} \pm 4.1^{\circ}, 15.7^{\circ} \pm 3.9^{\circ}, 16.0^{\circ} \pm 4.0^{\circ}$, and $16.1^{\circ} \pm 4.2^{\circ}$, respectively. Here, all comparisons revealed significant differences except among the 3rd, 4th, and 5 th visits.

\section{Resting calcaneal stance position}

RCSP measurements at baseline, 2nd, 3rd, 4th, and 5th visits were $-8.3^{\circ} \pm 5.6^{\circ},-2.2^{\circ} \pm 2.5^{\circ},-1.7^{\circ} \pm 2.0^{\circ},-1.6^{\circ} \pm 3.2^{\circ}$, and $-1.5^{\circ} \pm 2.7^{\circ}$, respectively. The $2 \mathrm{nd}, 3 \mathrm{rd}, 4$ th, and 5 th
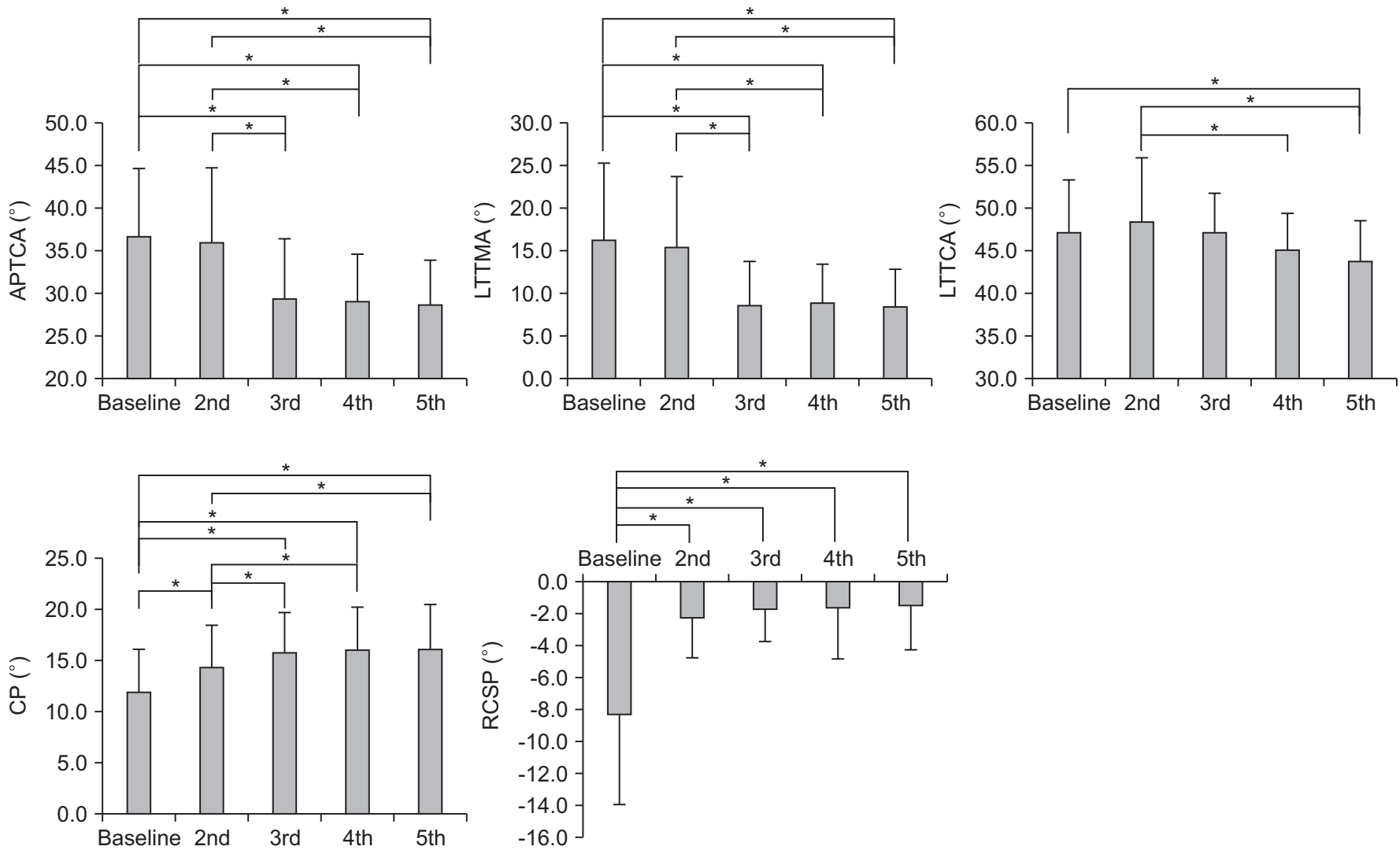

Fig. 2. Serial changes in anteroposterior talocalcaneal angle (APTCA), lateral talocalcaneal angle (LTTCA), lateral talometatarsal angle (LTTMA), calcaneal pitch (CP), and resting calcaneal stance position (RCSP) over time. ${ }^{*} \mathrm{p}<0.05$; $2 \mathrm{nd}$, $3 \mathrm{rd}$, 4th, and 5th correspond to evaluations conducted at 12-18, 24-30, 36-42, and $\geq 48$ months after rigid foot orthosis application (i.e., baseline). 
measurements represented statistically significant improvements over the baseline.

\section{DISCUSSION}

The present study demonstrated long-term (i.e., $>4$ years) trends in changes of positions of the talus and the calcaneus following the application of a custom-made RFO. We measured four radiologic indicators to evaluate the alignment of the mid to hindfoot, with the aim to increase reliability of the results and evaluate relationships between various parts of the foot. The CP known to be only affected by the calcaneal factor exhibited the greatest significant increase between the baseline and 2nd visits. Although the 3rd visit also represented a significant change relative to the 2nd visit, the difference was not greater than that observed during the first interval. A similarly significant difference in RCSP was also observed between the baseline and the 2nd visit. However, no significant differences were observed between other visits. These findings might be due to the fact that the medial side of the calcaneus is the primary target of the custommade RFO. Therefore, changes in this factor are the most obvious in the beginning.

We also observed that in contrast with significant increases in indices associated only with the calcaneus, the LTTCA tended to increase only from the baseline to the 2nd visit. This outcome might be due to the lack of calibration of the talus at baseline. However, this value decreased gradually over time and normalized after 4 years probably due to gradual talar inclination. Moreover, we did not observe a significant decrease in the APTCA from the baseline to the second visit whereas this value decreased significantly from the 2 nd to the 3 rd visit, indicating that talus changed mainly after the early calcaneal inclination between the 2nd and 3rd visits. Finally, the greatest change in the LTTMA value was observed between the 2nd and 3rd visits, further confirming that the greatest talar inclination occurred between these time points.

We further noted no significant changes in any of the measurements between the 3rd and 5th visits, although some tendencies were observed. Taken together, our findings indicate that most changes associated with RFO use occur soon after application. They are subsequently maintained. Furthermore, all findings indicate that a change in the talus occurs only after a change in the initial calcaneus inclination.

Compared with previous studies, we observed additional changes in radiographic indices after 2 years. Most radiographic indices exhibited significant changes within the first 2 years and a gradual plateau in the slope [9]. In a study of spontaneous changes in radiographic indices in planovalgus children, Park et al. [16] have reported that slope patterns tend to decrease beyond 10 years of age and plateau until skeletal maturity. In our study, the mean age at the beginning of RFO use was 9.79 years. Slope patterns of radiographic indices decreased after 2 years of rapid change. Our data combined with previous study findings suggest that the RFO should be worn for at least 2 years. Patient's age appears to be more important than the absolute wearing period. It is advisable to wear the orthosis until skeletal maturity is achieved unless a cavus deformity is observed during RFO use.

Our study had several limitations. First, it was limited by the lack of a control group. However, we were unable to enroll a control group given the clinical nature of this study and the fact that participants' parents did not consent to the inclusion of their children as controls. Second, we did not investigate pain. As noted above, many cases of flexible flat foot are asymptomatic. Because we did not observe symptoms in this study, we could not know whether improvements in radiologic indicators were associated with changes in symptoms. However, according to our clinical experience, patients with flexible feet usually complain of pain in the medial arch and dull pain in the ankle possibly due to excessive pressure of ligaments in soles and an inability to achieve proper force distribution. The pain may also be affected by excessive relaxation of the ligament due to eversion of the calcaneus and supination of the forefoot in the ankle joint. Furthermore, some children with flexible flat foot exhibited tightness in the Achilles tendon. Correction of these problems may improve pain in the affected foot. Future studies should evaluate foot pain in an outpatient clinical setting and the relationship between RFO use and pain.

In conclusion, results of this study indicate that custom-made RFO can initially lead to significant improvements in calcaneus-related radiographic indices followed by significant improvement in talus-related radiologic indices. The most significant improvements were observed within 26 months for calcaneus-related radiographic 
indices and after 18 months for talus-related radiologic indices which changed more gradually. Future studies should aim to conduct a randomized controlled trial involving a control group with intergroup comparisons for symptoms and radiologic indices.

\section{CONFLICT OF INTEREST}

No potential conflict of interest relevant to this article was reported.

\section{AUTHOR CONTRIBUTION}

Conceptualization: Bok SK, Kim BO, Park IS. Methodology: Youn KJ, Park IS. Formal analysis: Ahn SY. Funding acquisition: Bok SK, Park IS. Project administration: Youn KJ, Bok SK. Visualization: Youn KJ. Writing - original draft: Youn KJ, Bok SK. Writing - review and editing: Ahn SY, Bok SK. Approval of final manuscript: all authors.

\section{REFERENCES}

1. Harris EJ, Vanore JV, Thomas JL, Kravitz SR, Mendelson SA, Mendicino RW, et al. Diagnosis and treatment of pediatric flatfoot. J Foot Ankle Surg 2004;43:341-73.

2. Baroni MP, Sanchis GJ, de Assis SJ, dos Santos RG, Pereira SA, Sousa KG, et al. Factors associated with scoliosis in schoolchildren: a cross-sectional population-based study. J Epidemiol 2015;25:212-20.

3. Kuhn DR, Shibley NJ, Austin WM, Yochum TR. Radiographic evaluation of weight-bearing orthotics and their effect on flexible pes planus. J Manipulative Physiol Ther 1999;22:221-6.

4. Kim SB, Yoon K, Park HS, Kwak H, Ha NJ, Park JS. Radiologic measurement of flatfoot. J Korean Acad Rehabil Med 2000;24:995-1001.

5. Kothari A, Dixon PC, Stebbins J, Zavatsky AB, Theolo- gis T. The relationship between quality of life and foot function in children with flexible flatfeet. Gait Posture 2015;41:786-90.

6. Evans AM, Rome K. A Cochrane review of the evidence for non-surgical interventions for flexible pediatric flat feet. Eur J Phys Rehabil Med 2011;47:69-89.

7. Kulcu DG, Yavuzer G, Sarmer S, Ergin S. Immediate effects of silicone insoles on gait pattern in patients with flexible flatfoot. Foot Ankle Int 2007;28:1053-6.

8. Whitford D, Esterman A. A randomized controlled trial of two types of in-shoe orthoses in children with flexible excess pronation of the feet. Foot Ankle Int 2007;28:715-23.

9. Bok SK, Kim BO, Lim JH, Ahn SY. Effects of custommade rigid foot orthosis on pes planus in children over 6 years old. Ann Rehabil Med 2014;38:369-75.

10. Pfeiffer M, Kotz R, Ledl T, Hauser G, Sluga M. Prevalence of flat foot in preschool-aged children. Pediatrics 2006;118:634-9.

11. Lin CJ, Lai KA, Kuan TS, Chou YL. Correlating factors and clinical significance of flexible flatfoot in preschool children. J Pediatr Orthop 2001;21:378-82.

12. Volpon JB. Footprint analysis during the growth period. J Pediatr Orthop 1994;14:83-5.

13. Vanderwilde R, Staheli LT, Chew DE, Malagon V. Measurements on radiographs of the foot in normal infants and children. J Bone Joint Surg Am 1988;70:40715.

14. Blake RL. Inverted functional orthosis. J Am Podiatr Med Assoc 1986;76:275-6.

15. Blake RL, Ferguson H. Foot orthosis for the severe flatfoot in sports. J Am Podiatr Med Assoc 1991;81:549-55.

16. Park MS, Kwon SS, Lee SY, Lee KM, Kim TG, Chung CY. Spontaneous improvement of radiographic indices for idiopathic planovalgus with age. J Bone Joint Surg Am 2013;95:e193(1-8). 